\title{
ISSUES IN OPTIMIZING THE PERFORMANCE OF WIRELESS SENSOR NETWORKS
}

\author{
Rakesh.N $\mathbf{N}^{\mathbf{1}}$, Kayalvizhi Jayavel ${ }^{\mathbf{2}}$ \\ ${ }^{1}$ Department of Information Technology, SRM University, Chennai-603203 \\ ${ }^{2}$ Department of Information Technology, SRM University, Chennai-603203
}

\begin{abstract}
Wireless sensor networks have attracted most of the research efforts due to the latest advancements in the field of sensing, computing and communication. Wireless sensor networks provide endless opportunities as well they pose formidable challenges primarily pertaining to energy constraints to which sensor nodes are particularly subjected.Issues like data collection, load balancing ,battery depletion have to be addressed at the cost of optimization of wireless sensor networks. This paper provides the ongoing research activities and possible solutions to the problems
\end{abstract}

\section{INTRODUCTION}

Wireless sensor networks are a large number of small sensing battery powered nodes which gather information and communicate in a wireless fashion to the base station. The WSN contains nodes which can vary from a few to several thousand depending on the type of the application being deployed, where each node acts as a sensor. The topology of the WSNs can vary from a simple star network to an advanced multi-hop wireless mesh network. Propagation of data between the hops of the sensor network can be done either by routing or flooding. The fundamental task for wireless sensor network is data gathering which has became challenging factor due to the battery depletion, large number of nodes and certain other factors like hardware, operating system is responsible for it .Optimization of wireless sensor networks is possible only if we consider all the characteristics of Wireless sensor networks for resolving the issues which optimize the performance of the wireless sensor networks on the whole.

\subsection{Characteristics of a Wireless Sensor Network}

The fundamental characteristics of a Wireless sensor network include:

- Energy consumption constraints for nodes using batteries:

The nodes of wireless sensor networks are battery powered and in many cases no replacement of batteries can be done once deployed to the user .So, energy consumption has to be efficiently managed to increase the life time of the sensor networks [8].

- Mobility of nodes:

In Wireless sensor networks nodes can change their location with time. Mobility of nodes help us from the problems regarding the constraints of network connectivity.
- Communication failures:

Communication is done by either flooding or routing. Communication devices mainly used in wireless sensor networks are the radio transceivers. Radio transceivers are the devices comprising of both transmitter and receiver to work within the radio frequency range. Communication failures occur due to battery depletion.

- Heterogeneity of nodes:

Wireless sensor networks are provided with heterogeneity of nodes .For example three primary types of hardware heterogeneities are Computational heterogeneity in which some nodes are provided with extra computational abilities. Link heterogeneity, where some nodes are provided with long-distance highly reliable communication links . Energy heterogeneity where nodes have unlimited energy resources.

- Scalability to large scale of deployment

Wireless sensor networks are scalable on a large scale. If sensor nodes are being deployed then battery cannot be changed and even providing maintenance cannot be possible.

Issues that avoid the optimization of a wireless sensor network are

\subsection{Data Collection}

The primary task of sensor nodes is systematic collection of data and transmit the gathered data in a wireless fashion to the base station i.e., Data collection. In wireless sensor networks nodes can communicate if and only if they are in common communication range. The time for which the nodes are in contact is defined as the contact time. The contact area is defined as the region where that node can possibly be in contact with other nodes. They are mainly three phases in data collection they are discovery, data transfer and routing. 
Discovery is the first phase in collecting data in The primary task of discovery protocols is to detect contacts as soon as they happen, and with a low energy expenditure. The main aim of discovery phase is to try to maximize both the number of detected contacts, and the residual contact time, while minimizing the energy consumption.

Data transfer is the second phase in the data collection. The task of data transfer protocols is to get the most out of the residual contact time, that is, to maximize the throughput in message exchange between nodes which are in contact while minimizing the energy consumption.

Routing is the last phase in the data collection. It is possible only when we have large number of nodes i.e., when the density of the network is enough to allow multi-hop routes .The task of routing is to find the best multi-hop paths in terms of both delivery ratio and low energy consumption to avoid battery depletion.

Wireless sensor network aims to collect sensor readings at predefined Sinks for analysis and processing. Sinks are static sources which are sometimes given mobility constraints. Sensor generate their data, then data is sent to one or more sinks . Each sensor measurement arrives at the sink without any changes.. Constrained mobile sink has limited communication time to collect data from sensor nodes deployed randomly which poses some significant challenges. Sensor near the sink depletes their battery power compared to than those far apart this will cause Non-uniform energy consumption. Non-uniform energy consumption causes degraded network performance and shortens network lifetime. If all the sensors run out of energy the sink will be isolated from the network, so the entire network fails then data gathering is said to be failure.

\subsubsection{Solution:}

This issues can be solved by Clustering .Clustering is the method by which sensor nodes in a network organize themselves into hierarchical structures. By doing this, sensor nodes can use the scarce network resources such as radio resource, battery power more efficiently. Within a particular cluster, data aggregation and fusion are performed at cluster- head to reduce the amount of data transmitting to the base station. Cluster formation is usually based on remaining energy of sensor nodes and sensor's proximity to cluster-head . Non cluster-head nodes choose their cluster-head right after deployment and transmit data to the cluster-head.

\subsubsection{Active Research Work:}

In late numerous clustering algorithms have been proposed . .We have to choose an algorithm for which the energy consumption ,and the lifetime of the whole network will be optimized.

\subsection{Density Control:}

The number of deployed sensor nodes can be really large, so the most important aspect is density control. In a dense network, many sensor nodes can work on the same region, generating redundant data. This undesirable because it leads to more network traffic and increases the energy consumption.

\subsubsection{Solution:}

The density control mechanism adopted prevents sensor nodes to work in the same region at the same time and ensures monitored area coverage. Density control is an important aspect when we have dense WSNs. Density control mechanism role is to manage the redundancy, keeping active only a minimum set of sensor nodes at a certain time.

\subsubsection{Active Research Work:}

Different density control mechanisms are being proposed for avoiding redundancy. Mainly various centralized and distributed techniques have been proposed for density control. Centralized heuristic is proposed, to divide the set of sensor nodes in mutually exclusive subsets where each subset covers the sensing area. Whereas in distributed density control algorithm idea is to make the sensor nodes temporarily inactive, i.e. in sleep mode, when they are not essential to guarantee coverage and connectivity

\subsection{Sink Mobility:}

In a WSN architecture, the sink is a special node which function is to gather the collected data and send it outside the network. We does not consider a fixed sink. Nevertheless, since a WSN is expected to have a large number of sensor nodes, it could be impossible to move a sink node to each sensor communication range.

\subsubsection{Solution:}

Cluster-based approach communication will make it easy to solve this problem without sink mobility. Since these networks will probably have more than one cluster, we need to efficient routes to sink tour among these clusters.

\subsubsection{Active Research Work:}

In late many clustering algorithms are being proposed for efficient data collection for a fixed sink.Selecting an algorithm with clustering based approach and efficient in terms of throughput, other performance parameters compared to other clustering algorithms will help to optimize the wireless sensor networks.

\subsection{Load Balancing:}

Wireless sensor networks have received more attention in the recent years due to the advancements of research efforts. In sensor networks they are large number of nodes, managing such 
large number of nodes have to be done properly due to the energy constraints that are imposed on sensor networks. Due to the increase in load some sensor nodes may run out of energy before communicating with sink which will lead to network failure

\subsubsection{Solution:}

An algorithm that can optimize the load and also performance parameters like throughput have to be proposed in such a way to increase the span of network life time and to avoid network failures

\subsubsection{Active Research Work:}

Few algorithms based on Load balanced clustering algorithms have been proposed in a way to optimize the load, energy ,size.For example DSBCA(Distributed Self-Organization For Wireless Sensor Networks) algorithm takes all the aspects such as energy level of nodes, distance from the sink for electing a cluster head in every cycle from where data is then forwarded to sink. DSBCA also has restrictions on the maximum size of the cluster.

\subsection{Security:}

Security in wireless sensor networks is the most important factor as sensor networks being used in the real-time and critical applications like burglar alarms, surveillance, in airports and hospitals. Sensor networks are the recent research trend in which new algorithms, technologies are being proposed. Sensor networks are in the early stage of development, by integrating security in the wireless sensor networks they provide confidentiality and protection of data. Different types of problems considering the security aspects in sensor networks are and changing the routing information, passive information gathering, node subversion, sinkhole attacks, DOS attacks and jamming.

\subsubsection{Solution:}

The possible solution is to design security features which can be integrated into a routing protocol, cryptography for sensor networks using a new symmetric key, designing data aggregation protocols along with some security constraints, designing intruder detection systems.

\subsubsection{Active Research Work:}

Security is in the early stages in terms of wireless sensor network. Research work was being done to integrate the security systems in to wireless sensor networks to provide authentication, confidentiality and protection of data

\subsection{Synchronization:}

Synchronization is one of the vital service in sensor networks. The main task of Synchronization in a sensor network is to provide a common time range for local clocks of nodes in the network. Synchronization will help the sensor system to process and analyze the data correctly and predict future system behavior. In data aggregation the data collected from different nodes are combined into a meaningful result. If synchronization is not available for sensor nodes then the data estimation will be not be appropriate. So data estimation can be achieved only with synchronization

\subsubsection{Active Research work:}

Research work include improving the existing synchronization protocols like Light Weight Tree Based Synchronization(LTS) and Reference Broadcast Synchronization(RBS) .Radio communication that uses multihop synchronization can be improved by building a new analytical model.

\subsection{WSN Communication Characteristics:}

Performance of wireless sensor networks depends on the of wireless communication. In sensor networks the wireless communication cannot be predicted due to the mobility of nodes ,power consumption constraints for nodes using batteries ,ability to cope with node failures, communication failures.

\subsubsection{Solution:}

To cope with the flaws of communication characteristics of wireless sensor network we must design low battery consuming communication systems and routing algorithms that can optimize the performance of sensor networks that can avoid communication failures and increase network life time.There is also a need to design a new architecture for integrated wireless sensor systems .

\subsection{Transport Layer Issues:}

$\mathrm{Tcp} / \mathrm{Ip}$ is the most deployed protocol in the internet .But it is highly unsuitable for wirless sensor networks for its communication over internet due to overhead, congestion control .Also sensor networks are used in real time applications using tcp with wirless sensor networks may cause delay due to overhead, large size of the tcp header. In sensor networks data loss occurs when the data is transferred from sink to source which is undesirable because whenever sensor gathers information and transfers it to the sink which in turn requests the sensor node for sensed information

\subsubsection{Active Research Work}

To overcome this flaws some transport protocols like 6lowpan are proposed for low power devices like sensor networks which can overcome some flaws of using tcp. In 6lowpan. It Separates control plane, data plane to overcome the overhead of using TCP . Existed protocols have not taken priority when routing. in to consideration which was essential because even the sensor nodes 
at different locations may have different priorities.Routing through multiple paths is under research.

\subsection{Network Layer Issues:}

Routing is the most important service in wireless sensor communication. Battery depletion indirectly affects the routing as it leads to network failure .If the number of nodes are more in wirless communication it would be impossible for the sink to communicate to every node in single hop.

\subsubsection{Solution:}

Several routing Protocols are being proposed for wireless sensor networks like LEACH(Low Energy Adaptive Cluster Hierarchy), TEEN(Threshold sensitive Energy Efficient sensor Network protocol), GEAR (Geographic and Energy Aware Routing), SAR (Sequential Assignment Routing),DSBCA (Distributed Self-Organization For Wireless Sensor Networks), SPIN(Sensor Protocols for Information negotiation)and others Selecting the best routing protocol suitable to the requirements.

\subsection{Deployment:}

Sensor nodes are deployed in a large scale over a particular region or a sensor field in a real-time environment. sensor nodes being deployed in real-time environment will provide wrong readings due to the battery depletion as no battery replacement is possible after deployment in wirless sensor networks .So issues affecting sensor nodes must be detected for avoiding the data loss. Also, concurrent transmission attempts done due to the deployment of sensor nodes will lead to congestion.

\subsubsection{Active Research Work:}

Research work includes improving the range of the radio when deployed in various real time environment. Wrong sensor readings have to be detected as soon as possible to decrease latency and congestion.

\section{CONCLUSIONS}

Wireless Sensor Networks created a wide range of oppurtunities as well as challenges that have to be addressed at the cost of optimization. In this paper we have identified a list of issues associated with Wireless Sensor Networks. We have discussed some solutions and active research work pertaining to these issues

\section{REFERENCES}

[1]. "A Review of Current Routing Protocols for Adhoc Wireless Mobile Networks", IEEE Personal Communications, April 1999, pp 46-55.

[2]. I. Akylidiz, W. Su, Sankarasubramaniam, and E.Cayrici, "A survey on sensor networks", IEEE Communications Magazine, Volume: 40 Issue: 8, August 2002, pp.102-114.
[3]. Q. Jiang and D.Manivannan, "Routing Protocols for Sensor Networks", In proceedings of Consumer Communications and Networking Conference, 5-8 January, pp 93-98.

[4]. Ning $\mathrm{Xu}$, "A survey of Sensor Network and its Applications",http://enl.usc.edu/ ningxu/papersurvey.pdf.

[5]. K. Akkaya and M. Younis, "A survey of Routing Protocols in Wireless Sensor Networks", Elsevier Ad Hoc Network Journal, 2005, pp 325-349.

[6]. D.Culler, D.Estrin and M.Srivastava, "Overview of Sensor Networks", IEEE Computer Society, August 2004

[7]. Ying Liao, Huan Qi, and Weiqun Li, "Load-Balanced Clustering Algorithm With Distributed Self-Organization for Wireless Sensor Networks" IEEE SENSORS JOURNAL, VOL. 13, NO. 5, MAY 2013.

[8]. Daniele Puccinelli and Martin Haenggi "Wireless Sensor Networks:Applications and Challenges of Ubiquitous Sensing" IEEE CIRCUITS AND SYSTEMS 2005.

[9]. "Data Collection in Wireless Sensor Networks with Mobile Elements: A Survey" Mario Di Francesco and Sajal K. DasCrewman, The University Of Texas At Arlington And GIUSEPPE ANASTASI, University of Pisa.

[10]. "Issues in Wireless Sensor Networks"Gowrishankar.S , T.G.Basavaraju, Manjaiah D.H , Subir Kumar Sarkar Proceedings of the World Congress on Engineering 2008 Vol IWCE 2008, July 2 - 4, 2008, London, U.K. 